\title{
Glow Discharging Your Grids Before Plunging Your Sample is not Necessary
}

\author{
Dennis Thomas, Katarina Meze, Chou Tsung Han and Dexter Adams \\ Cold Spring Harbor Laboratory, Cold Spring Harbor, New York, United States
}

\begin{abstract}
The carbon surfaces of the grids we typically use tend to be hydrophobic causing difficulty in getting an aqueous sample to spread uniformly over the grid. Glow discharging the grids before use imparts a surface charge to the carbon which promotes even spreading of the sample over the grid. One drawback is our proteins of interest adhere to the charged surface, a) reducing the number of particles present in the holes of quantifoilsand C-flats and b) the buildup of particles on the carbon appears to increase the ice thickness in the holes. We show here that pretreatment of the grids by washing with ethyl acetate and then applying the sample to the grids has nearly the same behavior as if glow discharged. Having detergent present improves sample spreading but is not necessary.. This approach maybe of use for samples which stick heavily to carbon and are hard to get into holes using glow discharge or which are only available in limited concentrations.
\end{abstract}

The experiment: Quantifoil grids were used for the experiment. R1.2/R1.3 on molybdenum or copper,, 0.6/1 on copper. Before use grids held in forceps were dipped into ethyl acetate for about 10 seconds then dried on filter paper. Ethyl acetate has been used as a solvent for the plastics, such as triafol, used for making holey grids. $4 \mathrm{ul}$ of sample was applied to each grid and grids were blotted using a Leica GP2 plunger using the sensor blotting function. The one sided blotting of the grids in the Leica GP2 allowed observation of the process.

In parallel grids were glow discharged in a Pelco Easy Glow device using air at $0.42 \mathrm{mBar}, 15 \mathrm{~mA}$ current, for 35 seconds. The same blotting procedures were used. Some glow discharged examples were blotted using a vitrobot. Protein samples used include Apo ferritin, membrane receptor, Disraeli/RNA complexes, Argonaut, beta galactosidase. The method has been found to be useful for numerous samples.

CryoEM data was acquired on a Titan G3 microscope using either a K2 or K3 camera. EPU software was used for acquisition.

Results: The sample applied to untreated grids beads as on parafilm see figure 1 panel A. Surprisingly samples without detergent applied to EtOAc washed grids behaved much like when applied to glow discharged grids figure 1 panel $\mathbf{B}$ and C compare to D. Samples with detergent behaved best spreading and blotting uniformly over the grid Figure 2. Grid maps of several samples are shown in figure 3.

Discussion: This method has the same caveats as other techniques for preparing samples for cryoEM, i.e. success and behavior is sample dependent. However it can clearly help where samples adhere strongly to the carbon after glow discharging. This can be important when sample concentration is limiting. Also the attraction of the sample to the charged carbon surface can make the ice in the holes thicker.

References

Preparation of macromolecular complexes for cryo-electron microscopy Robert A Grassucci, Derek J Taylor, and Joachim Frank, Nat Protoc. 2007 ; 2(12): 3239-3246 